\title{
Clinical implications of the global multidrug-resistant tuberculosis epidemic
}

\author{
Authors: Kartik Kumar ${ }^{\mathrm{A}}$ and Ibrahim Abubakar ${ }^{\mathrm{B}}$
}

\begin{abstract}
Multidrug-resistant tuberculosis (MDR TB) is a significant threat to global health estimated to account for nearly half a million new cases and over 200,000 deaths in 2013 . The number of MDR TB cases in the UK has risen over the last 15 years, with ever more complex clinical cases and associated challenging public health and societal implications. In this review, we provide an overview of the epidemiology of MDR TB globally and in the UK, outline the clinical management of MDR TB and summarise recent advances in diagnostics and prospects for new treatment.
\end{abstract}

KEYWORDS: Multidrug-resistant tuberculosis, epidemiology, diagnostics, treatment

\section{Introduction}

The significant threat of the global multidrug-resistant tuberculosis (MDR TB) epidemic has been described as a crisis in the World Health Organization's (WHO's) End TB strategy. ${ }^{1}$ MDR TB is defined as tuberculosis (TB) caused by Mycobacterium tuberculosis isolates that are resistant to at least both rifampicin and isoniazid. ${ }^{2}$ The WHO's Global tuberculosis report supplement in 2014 estimated that MDR TB accounts for 3.5\% (95\% confidence interval (CI) 2.2-4.7\%) of all new cases of TB and $20.5 \%$ (95\% CI 13.6-27.5\%) of TB cases among individuals previously treated for the disease. ${ }^{3}$ There were approximately 480,000 new cases of MDR TB globally in 2013, with an estimated 210,000 deaths attributable to MDR TB. The highest levels of MDR TB are in Eastern European and Asian countries, with more than half the global burden of MDR TB being located in India, China and Russia. ${ }^{4}$ Additional cause for concern is the emergence of extensively drug-resistant TB (XDR TB), which is defined as tuberculosis caused by $M$ tuberculosis isolates that are resistant to rifampicin, isoniazid, any

Authors: ${ }^{\text {A }}$ academic foundation year 2 doctor, Centre for Infectious Disease Epidemiology, Research Department of Infection and Population Health, University College London, London, UK, and The Whittington Hospital NHS Trust, Whittington Health, London, UK; ${ }^{\text {B }}$ professor of infectious disease epidemiology, Centre for Infectious Disease Epidemiology, University College London, London, UK, and MRC Clinical Trials Unit, University College London, London, UK fluoroquinolones and at least one of the second-line injectable drugs (capreomycin, kanamycin and amikacin). ${ }^{2}$

Here we provide an overview of the importance of MDR TB, its epidemiology, clinical implications, potential ways of addressing the disease and prospects for new diagnostics and treatment.

\section{Epidemiology \\ Global epidemiology of TB}

Unfortunately, data on drug resistance are limited by a number of factors, including lack of facilities for testing and the limited availability of routine surveillance that only occurs in approximately $50 \%$ of countries. Of the 144 countries known to be collecting data on TB drug resistance, 72 have continuous surveillance systems in place that are based on drug susceptibility testing (DST) of all TB patients. The other 72 countries undertake special surveys of representative patient samples every 3-5 years, with this approach being commonest in settings where resources are limited. Out of the 36 high TB and high MDR TB countries, 10 have the former surveillance approach, while 26 have adopted the latter. Molecular technologies are increasingly being used for surveillance: examples include GenoType ${ }^{\circledR}$ MTBDRplus (Hain Lifescience, Nehren, Germany) and Xpert ${ }^{\circledR}$ MTB/RIF (Cepheid Inc, Sunnyvale, CA, USA).

Among newly diagnosed cases of TB, the highest levels of MDR TB have been found in Belarus (35.2\% in 2013), Kyrgyzstan (26.4\% in 2011) and Kazakhstan (25.2\% in 2013). Among previously treated cases, the levels are highest in the Republic of Moldova (62.3\% in 2012), Uzbekistan $(62.0 \%$ in 2011) and Tajikistan (56.0\% in 2012).

The total number of formally notified cases globally was 136,412 cases of MDR TB or rifampicin-resistant TB (RR TB) in 2013, substantially lower than the estimated 300,000 (range 230,000-380,000) MDR TB cases among pulmonary TB cases and 480,000 (range 350,000-610,000) incident MDR TB cases among all cases. The majority of these reported cases arose from Europe $(45,136)$, India $(35,385)$ and South Africa $(26,023)$. The higher number of MDR TB cases in 2013 compared to 2012 was due to the increase in the number of cases in India (increased from 21,498 to 35,385), Uzbekistan (increased from 2,233 to 5,751) and Ukraine (increased from 7,615 to 10,585). Between 2012 and 2013, MDR TB and RR TB notifications increased in the Democratic Republic of Congo, Myanmar, Tajikistan and Uzbekistan while notifications decreased in Belarus, Bulgaria, Estonia, Kazakhstan, Kyrgyzstan, Latvia, 
Lithuania and the Russian Federation. China reported 4,183 cases of MDR TB/RR TB of its estimated 54,000 MDR TB cases in 2013. In the same year, the Russian Federation reported 33\% of its estimated number of MDR TB cases.

Globally, 48\% of MDR TB patients from 2011 successfully completed treatment while $25 \%$ were either lost to follow-up or had no information available about their final outcome. The highest success rates were in the Eastern Mediterranean (64\%), while the lowest were in the European and African regions (less than 50\%). The highest treatment failure rate was in the European region (15\%) while the highest death rate was in South-East Asia (21\%). ${ }^{4}$

\section{Epidemiology of TB in the UK}

In 2013, there were 7,892 cases of TB notified in the UK with an incidence of $12.3 / 100,000 .{ }^{5}$ The majority of these cases (73\%) occurred in non-UK born individuals. Generally, however, the number of TB cases has fallen by $11.6 \%$ over the preceding two years owing to a small decrease in the number and rate of cases among the non-UK born population. TB continues to remain a health problem linked to socioeconomic status: $70 \%$ of TB cases lived in the $40 \%$ most deprived areas in the country; almost half of the cases were unemployed. Geographically, the greatest number of cases occurred in London, which accounted for $37.8 \%(2,985 / 7,892)$ of all TB cases with an incidence of $35.5 / 100,000$ (95\% CI 34.2-36.8). The most common countries of origin for non-UK born TB cases out of the 5,415 cases with a known foreign place of birth were India $(1,615)$, Pakistan $(1,103)$ and Somalia (292).

There was more isoniazid resistance among non-UK born cases than among UK-born cases $(8.0 \%, 267 / 3,332$ versus $4.6 \%, 52 / 1,127)$ in 2013 . The greatest number of cases of isoniazid-resistant TB occurred in people born in India, Pakistan and Somalia, while the greatest proportion of TB cases with isoniazid resistance occurred in those born in Ireland, Lithuania, Ukraine and Eritrea. There is an ongoing outbreak of isoniazid resistance in London (cluster E1244: strain type 424332431515321236423-52) which has affected mainly White and Black-Caribbean UK-born cases with social risk factors. Isoniazid resistance was more common in those with a previous history of TB among both UKborn cases $(5.3 \%, 4 / 75$ versus $4.7 \%, 47 / 1,008)$ and non-UK born cases $(10.7 \%, 18 / 169$ versus $7.9 \%, 241 / 3,052)$. Social risk factors such as homelessness, imprisonment and misuse of drugs or alcohol were associated with isoniazid resistance, which occurred in $15.3 \%(28 / 183)$ of those reporting drug misuse and $15.4 \%$ (23/149) of those reporting imprisonment.

Of all notified cases of TB in 2013, 1.6\% (74 cases) had MDR TB. The absolute number of cases of MDR TB has increased almost three-fold ( 86 cases in $2011 ; 82$ cases in 2012) compared to figures a decade ago. In total, $87.3 \%$ of the UK MDR TB cases in 2013 arose in people born outside of the UK. While the highest absolute number of cases of MDR TB arose from India, Pakistan and Somalia, the highest proportion of cases arose from Ukraine, Lithuania, Latvia and Sierra Leone. MDR TB was more common in males than in females $(1.7 \%, 48 / 2,764$ versus $1.4 \%, 26 / 1,842)$ and in the $15-44$-year-old age group $(2.1 \%, 61 / 2,931)$. A greater proportion of cases of MDR TB occurred in those with a previous history of TB than in those who had not previously had the disease $(4.0 \%, 10 / 250$ versus $1.4 \%, 59 / 4,128)$.

Within the MDR TB population group, $17.4 \%$ (12/69) of those who were tested for resistance against the four firstline drugs were resistant to all four of them. Half of MDR TB cases were resistant to at least one injectable agent. Those with resistance to an injectable agent were likely to be born in EU/ EEA countries $(58.3 \%, 7 / 12)$ while those with resistance to a fluoroquinolone were likely to be born outside the EU/EEA $(72.7 \%, 8 / 11)$. The proportion of MDR TB cases resistant to injectable agents rose from $2.1 \%$ in 2004 to $19.8 \%$ in 2012 . This has been attributed at least in part to the rise in the number and proportion of such cases arising from Lithuania between 2004 and 2013. Additionally, between 2010 and 2013, resistance to injectable agents was seen in all MDR TB cases from Russia as well as in at least half of MDR TB cases from China and Latvia. The majority of cases of MDR TB with fluoroquinolone resistance within this same time period arose among people born in India $(45.5 \%, 20 / 44){ }^{5}$

\section{Management of MDR TB}

\section{Diagnosing drug resistance}

Phenotypic drug susceptibility testing (DST) or rapid molecular diagnostics are required to make a diagnosis of drug-resistant TB. ${ }^{6}$ Conventional methods of DST and culture take a significant amount of time to yield results regarding mycobacterial growth and drug resistance. This runs the risk of patients being treated with incorrect drugs in the interim, thus permitting further spread of drug-resistant strains and promoting the selection of strains with even greater resistance (ie extensively drug-resistant TB). Rapid diagnosis may improve prognosis and prevent further resistance emerging and spreading. The higher rates of drug-resistant TB in particular subpopulations in the UK, such as those from hard to reach groups and migrants from specific countries, means that clinicians should order rapid tests when a diagnosis of $\mathrm{TB}$ is suspected in individuals from these populations. The results of DST to different drugs can vary in accuracy and reproducibility. DST to rifampicin, isoniazid, fluoroquinolones and second-line injectable drugs are sufficiently accurate and reproducible, such that the results of their DSTs can be used to tailor individualised treatment regimens. Similarly reasonably reliable molecular tests for rifampicin and isoniazid resistance endorsed by the WHO are commercially available.

\section{Treatment of MDR TB}

Treating MDR TB can be complex and expensive due to the number of antibiotics required, the potential for serious adverse effects and the possibility of interactions with other medications. WHO guidance states that there are various general principles to consider when treating MDR TB. The full details of these principles can be found in the $2014 \mathrm{WHO}$ publication Companion handbook to the WHO guidelines for the programmatic management of drug-resistant tuberculosis. ${ }^{6}$ In brief, these are: use at least four effective antibiotics in the intensive phase which should last eight months (including pyrazinamide and an injectable); avoid toxic agents; use more antibiotics in more drug-resistant cases; use ambulatory care and administer the drugs with directly observed therapy; and 


\section{Table 1. List of WHO-approved drugs for treating TB. ${ }^{6}$}

\author{
Group \\ 1: First-line oral agents \\ 2: Injectable anti-TB drugs \\ 3: Fluoroquinolones \\ 4: Oral bacteriostatic second-line anti-TB drugs \\ 5: Anti-TB drugs with limited data on efficacy and/or \\ long-term safety in the treatment of drug-resistant TB
}

\section{Drugs}

Isoniazid, rifampicin, ethambutol, pyrazinamide, rifabutin, rifapentine

Streptomycin, kanamycin, amikacin, capreomycin

Levofloxacin, moxifloxacin, gatifloxacin

Ethionamide, prothionamide, cycloserine, terizidone para-aminosalicylic acid, para-aminosalicylate sodium

Bedaquiline, delamanid, linezolid, clofazimine, amoxicillin/clavulanate, imipenem/cilastatin, meropenem, high-dose isoniazid, thioacetazone, clarithromycin

$\mathrm{TB}=$ tuberculosis; $\mathrm{WHO}=$ World Health Organization .

co-administer treatment with anti-retroviral drugs in patients who have HIV co-infection. The specific regimen for a patient will depend on their clinical history, DST results and contact history. The groups of drugs approved by WHO for treating TB are listed in Table $1 .^{6}$

The WHO advises caution against creating individualised regimens for patients based on DSTs to pyrazinamide, ethambutol, streptomycin, group-4 drugs and group-5 drugs, owing to problems with accuracy and reproducibility. The increasing occurrence of fluoroquinolone and injectable anti-TB drug resistance among MDR TB cases in the UK and the countries of origin of these patients should be taken into account when planning treatment regimens.

\section{Duration of treatment}

The intensive phase of MDR TB treatment refers to the length of time that patients receive injectable anti-TB drugs. WHO recommends that most MDR TB patients should receive the intensive phase of treatment for eight months. ${ }^{6}$ Response to treatment is suggested by smear conversion and culture conversion. Other factors suggesting successful response to treatment include improvement or resolution of symptoms and pulmonary lesions on radiography. For those patients that do not undergo conversion by the eighth month, failure of treatment needs to be considered. The total duration of treatment depends on whether the patient has previously been treated for MDR TB. For those patients who have been newly diagnosed, treatment for a total of 20 months is recommended. This may be modified according to the individual patient's response to therapy. In those who have previously been treated with the MDR TB regimen, treatment for a minimum of 24 months is recommended.

\section{Extrapulmonary MDR TB}

Most cases of extrapulmonary MDR TB can be treated in the same way as pulmonary MDR TB. ${ }^{6}$ However in central nervous system (CNS) MDR TB, the ability of drugs to penetrate the CNS needs to be taken into consideration. Isoniazid, pyrazinamide, prothionamide/ethionamide and cycloserine are able to penetrate the CNS well, while several other drugs have poor penetration, variable penetration or data on their use in CNS MDR TB are currently lacking.

\section{Surgical intervention in MDR TB}

Surgical resection of all or part of the lung has been shown to be safe and effective and thus may be considered as an adjunct to drug therapy. ${ }^{7}$ A minimum of two months of drug therapy should be administered prior to surgery. Surgery is not indicated if extensive bilateral disease is present. Surgery should take place when the patient's risk of morbidity or mortality is low: for example, when the disease is confined to a single lung. ${ }^{6}$

\section{Nutritional support}

Nutritional support should be provided to all MDR TB patients, as malnutrition causes worsening of and is worsened by MDR TB. ${ }^{8}$ This includes administering vitamin B6 (pyridoxine) in all MDR TB patients who are receiving isoniazid, linezolid, cycloserine or terizidone.

\section{Adverse effects of MDR TB therapies}

Recently, a guideline which succinctly describes the doses, adverse effects, interactions and monitoring requirements for each of the different drugs used in MDR TB has been published in the UK, providing a practical source of information for clinicians managing patients with the condition. ${ }^{9,10}$ Adverse effects range from common reactions, such as nausea, vomiting, diarrhoea and arthralgia, to a range of more severe reactions. Management of adverse effects depends on the severity of the side effect and which drug is responsible. Drugs used in the treatment of MDR TB also exhibit a range of drug interactions that further add to the complexity of managing the disease (see below). Patients with MDR TB should be managed by physicians with expertise in MDR TB treatment.

\section{Anti-retroviral therapy in MDR TB}

Use of anti-retroviral therapy (ART) improves outcomes for patients who are co-infected with HIV and MDR TB. ${ }^{11}$ WHO guidance states that ART is recommended for all patients with HIV and drug-resistant TB requiring second-line antiTB drugs, irrespective of CD4 cell-count, as early as possible (within the first eight weeks) following initiation of anti-TB treatment. ${ }^{12}$ Of clinical concern is the possibility of adverse effects arising from the overlapping toxicities of anti-TB drugs and ART. 
Drug-drug interactions may occur between second-line anti-TB drugs and ART agents. An online tool summarising all known interactions is available. ${ }^{13}$ The interaction between fluoroquinolones and didanosine may cause decreased fluroquinolone absorption. Clarithromycin has several interactions with protease inhibitors and non-nucleoside reverse transcriptase inhibitors.

\section{Technological advances in the management of MDR TB}

The labour-intensive and slow nature of conventional diagnostic tests to identify drug resistance in TB increases the risk of inappropriate TB drugs being prescribed and thus increases the likelihood of further drug resistance arising. Improving the efficacy and reliability of diagnostic tests will be fundamental to successfully identify, treat and thus reduce the global spread of MDR TB. Similarly, new treatment regimens with a shorter duration of administration and drugs that are less toxic will improve patient outcomes. Several technologies have been created to begin to address this problem.

\section{GeneXpert $^{\circledR}$}

The Xpert ${ }^{\circledR}$ MTB/RIF assay is an automated, real-time nucleic acid amplification assay that enables both the rapid detection of $M$ tuberculosis and the detection of mutations that may confer the bacterium with resistance to rifampicin. ${ }^{14}$ Sputum is added to a treatment reagent which liquefies and inactivates bacteria. The liquefied sputum is transferred to a cartridge which is loaded into the device for the assay which is based on real-time PCR amplification of the $r p o B$ gene target. A systematic review examining the diagnostic accuracy of molecular genetic tests for TB drug resistance has shown that GeneXpert ${ }^{\circledR}$ is sensitive, specific and cost-effective. ${ }^{15}$ It has been shown that a single, direct Xpert ${ }^{\circledR}$ MTB/RIF test detects $92.2 \%$ of culture positive patients, ${ }^{16}$ with the proportion detected increasing as the number of specimens that are tested increases. Similarly, the addition of second and third Xpert ${ }^{\circledR}$ MTB/RIF tests in smearnegative culture-positive patients increases the sensitivity of the test from 72.5 to 85.1 to $90.2 \%$ respectively. ${ }^{16}$ The test has been shown to have a sensitivity of $99.1 \%$ in detecting rifampicin resistance and specificity of $100 \%$ in patients with rifampicin susceptibility, while its rate of indeterminate results (3.7\%) was lower than the rate of culture contamination. ${ }^{16}$

In settings with low MDR TBR prevalence and where risk of MDR TB is low, there is not yet agreement about whether rifampicin resistance should be confirmed using a second specimen before treatment for MDR TB is initiated. ${ }^{17}$ It has further been suggested that the test's lower sensitivity in the context of less extensive disease may limit the utility of the test in low-incidence settings. ${ }^{18}$ Furthermore, the test has been shown to give false negative results in the presence of particular $r p o B$ mutations that are associated with low-level rifampicin resistance. ${ }^{19}$

\section{Line probe assays}

Line probe assays involve PCR amplification of $M$ tuberculosis DNA followed by hybridisation of labelled PCR products with specific oligonucleotide probes which are visualised as coloured bands on a strip. The process enables detection of $M$ tuberculosis and the presence of mutations that may confer resistance to drugs such as rifampicin. Mutations may be detected because the applied probes do not bind to the amplicon containing the mutation or they can be detected by using probes that are specific to a particular mutation. Line probe assays have been shown to have high sensitivity ( $>97 \%)$ and specificity (>99\%) for detecting rifampicin resistance in isolates from smear-positive sputum samples. Culture is still required for smear-negative specimens and DST is still required to confirm XDR TB; hence line probe assays are not currently considered a replacement for culture and DST. ${ }^{20}$ Benefits of this technique include the rapid speed with which rifampicin resistance can be detected, normally within 48 hours. ${ }^{21}$ Although the test has high sensitivity and specificity when used on culture isolates, it has lower sensitivity when used directly on clinical specimens. ${ }^{22}$ Additionally, it has been suggested that the reliability of the test in acting as a surrogate marker for MDR TB will depend on the prevalence of rifampicin resistance in the population. ${ }^{23}$

\section{Next-generation sequencing}

Next-generation sequencing (NGS) refers to technologies capable of very rapid high-throughput DNA sequencing, whereby millions of sequences are read in parallel. This offers huge potential to establish disease susceptibility and facilitate the development of targeted therapies. ${ }^{24}$ Studies have already illustrated a role for such technology in identifying drug resistance mutations in TB. ${ }^{25,26}$ NGS enables sequencing of entire coding regions and allows polymorphisms involved in drug resistance in TB to be further characterised. However, the discordance between phenotypic tests and NGS indicates that some mutations may not have an impact on drug susceptibility, so it is important to correlate new mutations with clinical response to drugs before determining what the effect of a particular mutation may be. ${ }^{25}$

\section{New drugs and regimens}

It was has been more than 40 years since the last new anti-TB drug, rifampicin, was introduced. ${ }^{27}$ Since then, the increasing incidence of MDR TB has led to a significant drive to develop new drugs that can tackle the growing threat of multidrug resistance. Two such drugs which have been licensed recently for MDR TB are bedaquiline and delamanid.

Bedaquiline (TMC207), a diarylquinoline, functions by inhibiting mycobacterial ATP synthase ${ }^{28}$ and has been developed to specifically treat MDR TB. It has been shown to be beneficial in phase-IIb trials ${ }^{29}$ but data from phase-III trials are currently awaited. Nevertheless, the US Food and Drug Administration granted bedaquiline accelerated approval in December $2012^{30}$ and subsequently the WHO issued interim guidance on the appropriate use of the drug in the treatment of MDR TB. ${ }^{31}$

Delamanid (OPC-67683) is a new nitro-hydro-imidazooxazole derivative that inhibits mycolic acid synthesis ${ }^{32}$ with encouraging trial results so far. ${ }^{33,34}$ It is currently under investigation in phase-III clinical trials as an adjunct to optimised background regimens for treating $\mathrm{MDR} \mathrm{TB}$, while other trials are currently evaluating the use of delamanid in children with MDR TB. In 2013, the European Medicine Agency recommended delamanid as a treatment option for $\mathrm{TB} .{ }^{35}$ 


\begin{tabular}{|c|c|}
\hline Trial & $\begin{array}{l}\text { New/repurposed drugs being } \\
\text { studied }\end{array}$ \\
\hline Delamanid phase III trial & Delamanid \\
\hline NExT trial & $\begin{array}{l}\text { Bedaquiline, linezolid, levofloxacin, } \\
\text { ethionamide/high-dose isoniazid, } \\
\text { pyrazinamide }\end{array}$ \\
\hline NiX-TB & $\begin{array}{l}\text { Pretomanid (PA-824), linezolid, } \\
\text { bedaquiline }\end{array}$ \\
\hline $\begin{array}{l}\text { STAND trial: PA-824/moxi/ } \\
\text { PZA (GATB NC-006) }\end{array}$ & $\begin{array}{l}\text { Pretomanid (PA-824), moxifloxacin, } \\
\text { pyrazinamide }\end{array}$ \\
\hline STREAM I Trial & Clofazamine \\
\hline STREAM II Trial & Clofazamine, bedaquilinine \\
\hline
\end{tabular}

A summary of phase III trials into new treatment regimens for MDR TB is provided in Table $2 .^{36}$

\section{Beyond MDR TB: the threat of XDR TB}

Treatment options are already severely limited for MDR TB. Affirmative action is therefore needed to halt the spread of drug resistance to avoid further spread of XDR TB which will be even more challenging to manage.

Cases of XDR TB were reported by 100 countries in 2013. Based on their combined data, an average of 9.0\% (95\% CI $6.5-11.5 \%)$ of MDR TB cases had XDR TB. The countries reporting the highest proportions of MDR TB cases with XDR TB were Lithuania (24.8\%), Kazakhstan (22.7\%) and Latvia $(21.7 \%)$. From the 1,269 patients in the global cohort of XDR TB patients in 2011: 22\% completed treatment; 35\% died; $10 \%$ failed treatment; and $33 \%$ were lost to follow-up or the treatment outcome was not evaluated. ${ }^{4}$ Data on treating XDR TB are limited. However it has been shown that a successful treatment outcome is more likely if at least six drugs are used in the intensive phase of treatment and four drugs are used in the continuation phase, and that outcomes are improved if latergeneration fluoroquinolones are used. ${ }^{37,38}$

\section{Future priorities}

The WHO has set out five priorities to eliminate TB: (i) reaching the 'missed' cases; (ii) addressing MDR TB as a crisis; (iii) accelerating the response to TB/HIV; (iv) increasing financing to close resource gaps; and (v) intensifying research and ensuring uptake of innovations.

Given the limited treatments available for MDR TB and the increasing threat of XDR TB, it is essential to address deficits in current practice. A critical step will be treating TB at its source and decreasing its transmission by ensuring that patients with active disease are rapidly identified, investigated and treated with the correct medications to which the strain of $M$ tuberculosis causing their disease is sensitive. Additionally it will be important to improve patient adherence to anti-TB medications and to take steps to decrease the likelihood of patients being lost to follow up. This should include ensuring that patients with TB have access to free medications and free laboratory testing. This will require significant financial investment into local healthcare systems. Wealthier countries will need to support resource-limited nations so that the appropriate infrastructure can be put in place to meet this need, which includes facilitating the use of new technologies that have been developed for rapid diagnosis of drug resistance in TB. Furthermore, new drugs that are currently in the pipeline need to be not only efficacious but also cost effective, so that the financial implications of treating TB do not create a barrier for developing countries. There needs to be increased provision for palliative care services in the context of MDR TB and XDR TB. Provision of integrated care services will be imperative in curbing the spread of the disease and above all the wider social determinants of TB need to be addressed. Most importantly, visionary leadership is needed if we are to tackle the global threat of MDR TB. ${ }^{39}$.

\section{Note}

This article was originally published in the 2015 Clinical Medicine supplement Horizons in Medicine 27. All articles in this supplement are available at www.clinmed.rcpjournal.org/content/15/Suppl_6

\section{References}

1 World Health Organisation. The end TB strategy. Global strategy and targets for tuberculosis prevention, care and control after 2015. Geneva: WHO, 2015. Available online at www.who.int/tb/ post2015_TBstrategy.pdf?ua=1 [Accessed 11 June 2015].

2 World Health Organisation. Definitions and reporting framework for tuberculosis - 2013 revision (updated December 2014. Geneva: WHO, 2014. Available online at http://apps.who.int/iris/ bitstream/10665/79199/1/9789241505345_eng.pdf [Accessed 11 June 2015].

3 World Health Organisation. Drug-resistant TB surveillance \& response. Supplement global tuberculosis report 2014. Geneva: WHO, 2014. Available online at http://apps.who.int/iris/bitstream/10665/137095/1/WHO_HQ_TB_2014.12_eng.pdf?ua=1 [Accessed 11 June 2015].

4 World Health Organisation. Global tuberculosis report 2014. Geneva: WHO, 2014. Available online at http://apps.who.int/iris/ bitstream/10665/137094/1/9789241564809_eng.pdf?ua=1 [Accessed 11 June 2015].

5 Public Health England. Tuberculosis in the UK: 2014 report. London: Public Health England, 2014. Available online at www.gov.uk/ government/uploads/system/uploads/attachment_data/file/360335/ TB_Annual_report_4_0_300914.pdf [Accessed 11 June 2015].

6 World Health Organisation. Companion handbook to the WHO guidelines for the programmatic management of drug-resistant tuberculosis. Geneva: WHO, 2014. Available online at http://apps.who.int/ iris/bitstream/10665/130918/1/9789241548809_eng.pdf?ua=1\&ua=1 [Accessed 11 June 2015].

7 Francis RS, Curwen MP. Major surgery for pulmonary tuberculosis: final report. Tubercle 1964;45(Suppl):5-79.

8 Sinclair D, Abba K, Grobler L, Sudarsanam TD. Nutritional supplements for people being treated for active tuberculosis. Cochrane Database Syst Rev 2011;(11):CD006086.

9 Keal JL, Capstick T, Ricketts WMR, Whitehead N, Kon OM. P97 Multi-drug resistant tuberculosis: The first UK guideline for treatment monitoring. Thorax 2013;68:A119.

10 Potter JL, Capstick Ricketts WMR T et al. A UK based resource to support the monitoring and safe use of anti-tuberculosis drugs and second line treatment of multidrugresistant tuberculosis. TB Drug Monographs, 2015. Available online www.tbdrugmonographs.co.uk [Accessed 11 June 2015]. 
11 Whalen C, Horsburgh CR, Hom D et al. Accelerated course of human immunodeficiency virus infection after tuberculosis. Am J Respir Crit Care Med 1995 Jan;151:129-35.

12 World Health Organization. Guidelines for the programmatic management of drug-resistant tuberculosis: 2011 update. Geneva: WHO, 2011. Available online at http://whqlibdoc.who.int/ publications/2011/9789241501583_eng.pdf [Accessed 11 June 2015].

13 HIV Drug Interactions (online website). Available online at www.hiv-druginteractions.org [Accessed 11 June 2015].

14 Weyer K, Mirzayev F, Migliori GB et al. Rapid molecular TB diagnosis: evidence, policy making and global implementation of Xpert MTB/RIF. Eur Respir J 2013;42:252-71.

15 Drobniewski F, Cooke M, Jordan J et al. Systematic review, metaanalysis and economic modelling of molecular diagnostic tests for antibiotic resistance in tuberculosis. Health Technol Assess 2015;19:1-188.

16 World Health Organization. Policy statement: automated real-time nucleic acid amplification technology for rapid and simultaneous detection of tuberculosis and rifampicin resistance: Xpert MTB/RIF system. Geneva: WHO, 2011. Available online at http://whqlibdoc.who.int/ publications/2011/9789241501545_eng.pdf [Accessed 11 June 2015].

17 Lange C, Abubakar I, Alffenaar JW et al. Management of patients with multidrug-resistant/extensively drug-resistant tuberculosis in Europe: a TBNET consensus statement. Eur Respir J 2014;44:23-63.

18 Sohn H, Aero AD, Menzies D et al. Xpert MTB/RIF testing in a low tuberculosis incidence, high-resource setting: limitations in accuracy and clinical impact. Clin Infect Dis 2014;58:970-6.

19 Somoskovi A, Deggim V, Ciardo D, Bloemberg GV. Diagnostic implications of inconsistent results obtained with the Xpert MTB/ Rif assay in detection of Mycobacterium tuberculosis isolates with an rpoB mutation associated with low-level rifampin resistance. J Clin Microbiol 2013;51:3127-9.

20 World Health Organisation. Policy statement: molecular line probe assays for rapid screening of patients at risk of multidrug-resistant tuberculosis (MDR-TB). Geneva: WHO, 2008. Available online at www.who.int/tb/ features_archive/policy_statement.pdf?ua=1 [Accessed 11 June 2015].

21 De Beenhouwer H, Lhiang Z, Jannes G et al. Rapid detection of rifampicin resistance in sputum and biopsy specimens from tuberculosis patients by PCR and line probe assay. Tuber Lung Dis 1995;76:425-30.

22 Morgan M, Kalantri S, Flores L, Pai M. A commercial line probe assay for the rapid detection of rifampicin resistance in Mycobacterium tuberculosis: a systematic review and metaanalysis. BMC Infect Dis 2005;5:62.

23 Traore H, Fissette K, Bastian I et al. Detection of rifampicin resistance in Mycobacterium tuberculosis isolates from diverse countries by a commercial line probe assay as an initial indicator of multidrug resistance. Int J Tuberc Lung Dis 2000;4:481-4.

24 Mardis ER. The impact of next-generation sequencing technology on genetics. Trends Genet 2008;24:133-41.

25 Walker TM, Kohl TA, Omar SV et al. Modernizing Medical Microbiology (MMM) Informatics Group. Whole-genome sequencing for prediction of Mycobacterium tuberculosis drug susceptibility and resistance: a retrospective cohort study. Lancet Infect Dis 2015;15:1193-202.
26 Takiff HE, Feo O. Clinical value of whole-genome sequencing of Mycobacterium tuberculosis. Lancet Infect Dis 2015;15:1077-90.

27 World Health Organisation. Frequently asked questions on bedaquiline. Geneva: WHO, 2015. Available online at www.who.int/tb/ challenges/mdr/bedaquilinefaqs/en/ [Accessed 11 June 2015].

28 Andries K, Verhasselt P, Guillemont J et al. A diarylquinoline drug active on the ATP synthase of Mycobacterium tuberculosis. Science 2005;307:223-7.

29 Diacon AH, Pym A, Grobusch MP et al. Multidrug-resistant tuberculosis and culture conversion with bedaquiline. $N$ Engl J Med 2014;371:723-32.

30 US Food and Drug Administration. FDA news release. Silver Spring, MD; FDA, 31 December 2012. Available online at www.fda.gov/ NewsEvents/Newsroom/PressAnnouncements/ucm333695.htm [Accessed 11 June 2015].

31 World Health Organisation. The use of bedaquiline in the treatment of multidrug-resistant tuberculosis. Interim policy guidance. Geneva: WHO, 2013. Available online at http://apps.who.int/iris/ bitstream/10665/84879/1/9789241505482_eng.pdf [Accessed 11 June 2015].

32 Kwon YS, Jeong BH, Koh WJ. Delamanid when other antituberculosis-treatment regimens failed due to resistance or tolerability. Expert Opin Pharmacother 2015;16:253-61.

33 Gler MT, Skripconoka V, Sanchez-Garavito E et al. Delamanid for multidrug-resistant pulmonary tuberculosis. $N$ Engl J Med 2012;366:2151-60

34 Diacon AH, Dawson R, Hanekom M et al. Early bactericidal activity of delamanid (OPC-67683) in smear-positive pulmonary tuberculosispatients. Int J Tuberc Lung Dis 2011;15:949-54.

35 European Medicines Agency. European Medicines Agency recommends two new treatment options for tuberculosis. London: European Medicines Agency, 2013. Available online at www.ema.europa.eu/ ema/index.jsp?curl=pages/news_and_events/news/2013/11/news_ detail_001972.jsp\&mid=WC0b01ac058004d5c1 [Accessed 11 June 2015].

36 RESIST-TB. DR-TB clinical trial progress report. Available online at www.resisttb.org/?page_id=1602 [Accessed 11 June 2015].

37 Falzon D, Gandhi N, Migliori GB et al. Resistance to fluoroquinolones and second-line injectable drugs: impact on multidrug-resistant TB outcomes. Eur Respir J 2013;42:156-68.

38 Jacobson KR, Tierney DB, Jeon CY et al. Treatment outcomes among patients with extensively drug-resistant tuberculosis: systematic review and meta-analysis. Clin Infect Dis 2010;51:6-14.

39 Abubakar I, Zignol M, Falzon D et al. Drug-resistant tuberculosis: time for visionary political leadership. Lancet Infect Dis 2013;13:529-39.

Address for correspondence: Prof I Abubakar, Centre for Infectious Disease Epidemiology, University College London, Mortimer Market Centre, Off Capper Street, London WC1E 6JB, UK.

Email: i.abubakar@ucl.ac.uk 